PESQUIMAT, Revista de la Fac.CC.MM. de la UNIVERSIDAD NACIONAL MAYOR DE SAN MARCOS

Vol.IV, $N^{\circ} 2$, pág.35-54, LIMA-PERÚ. Diciembre 2001

\title{
NULL CONTROLLABILITY FOR THE SEMILINEAR HEAT EQUATION IN UNBOUNDED DOMAINS
}

\author{
Silvano Bezerra de Menezes ${ }^{1}$ \& Victor R. Cabanillas ${ }^{2}$
}

\begin{abstract}
In this paper, we consider the null controllability problem for the semilinear heat equation in an unbounded domain $\Omega$ of $\mathbb{R}^{N}$ with Dirichlet boundary conditions. The control is assumed to be distributed along a subdomain $w$ such that the uncontrolled region $\Omega \backslash w$ is bounded. Using Carleman inequalities we first prove the null controllability of the linearized equation. Then, by a fixed point method, we obtain the main result for the semilinear case. This result asserts that, when the nonlinearity is globally Lipschitz, the system is null controllable.
\end{abstract}

Key words: null controllability, unbounded domain, approximate controllability, Carleman inequalities.

\section{INTRODUCTION AND MAIN RESULTS}

This paper is devoted to the study of the null controllability of the semilinear heat equation

$$
\left\{\begin{array}{l}
u_{t}-\Delta u+f(u)=h 1_{w} \text { in } \Omega \times(0, T) \\
u=0 \text { on } \Sigma=\partial \Omega \times(0, T) \\
u(x, 0)=u_{0}(x) \text { in } \Omega
\end{array}\right.
$$

where $\Omega$ is an open and unbounded set of $\mathbb{R}^{N}$ of class $C^{2}$ uniformly, with boundary $\partial \Omega$ (see Section 2 for a precise definition) and $w$ is an open and nonempty subset of $\Omega$. In (1.1) $u=u(x, t)$ is the state, $h=h(x, t)$ is the control function and $1_{w}$ denotes the characteristic function of the subset $w$.

\footnotetext{
${ }^{1}$ Departamento de Matemática, Universidade Federal do Pará, Brazil. Supported by the Alpha Project of EU "Modelling \& Engineering Mathematics" and CAPES-PICD.

${ }^{2}$ Facultad de Ciencias Naturales y Matemáticas - UNFV, Lima - Perú. Partially supported by CNPq, Brazil.
} 
Therefore, the control $h$ acts on the system thorough the subset $w$. We shall assume that $f$ is a real and globally Lipschitz function such that $f(0)=0$.

We also assume that the unbounded sets $\Omega$ and $w$ satisfy that

$$
\Omega \backslash w \text { is bounded. }
$$

According to this, the control acts on a large subset of $\Omega$ and only leaves a bounded subset of $\Omega$ without control. Therefore, the problem we are addressing is close to the classical one of controlling to zero the heat equation in bounded domains. We shall describe below the state of the art on this topic.

Let $u_{0} \in L^{2}(\Omega), \quad h \in L^{2}\left(0, T, L^{2}(\Omega)\right), \quad T>0$ and $f$ globally Lipschitz function such that $f(0)=0$. Then there exists a unique solution

of problem (1.1).

$$
u \in C\left([0, T], L^{2}(\Omega)\right) \cap L^{2}\left(0, T, H_{0}^{1}(\Omega)\right)
$$

The null controllability problem for (1.1) can be formulated as follows: Given $T>0$ and $u_{0} \in L^{2}(\Omega)$ to find a control $h \in L^{2}\left(0, T, L^{2}(\Omega)\right)$ such that the solution of (1.1) satisfies

$$
u(T)=0 \quad \text { in } \dot{\Omega}
$$

with an estimate of the form

$$
|h|_{L^{2}\left(0, T, L^{2}(\Omega)\right)} \leq c\left|u_{0}\right|_{L^{2}(\Omega)} \text {, for all } u_{0} \in L^{2}(\Omega) .
$$

Note that, in view of the condition $f(0)=0, u \equiv 0$ is an equilibrium solution of system (1.1) in the absence of control, i.e., with $h \equiv 0$. Thus, in the null controllability problem under consideration, we intend to drive the solution to the equilibrium in time $T$. Of course, if (1.3) is achieved, extending the control by zero for all $t \geq T$ we obtain a globally defined solution of (1.1) such that $u(t) \equiv 0$ for all $t \geq T$.

There is a large literature on the null controllability of heat equations in bounded domains. Let us briefly mention some of the existing works.

In the context of linear heat equations with time independent coefficients D.L. Russel [14] proved that the null controllability of the heat equation for all $T>0$ is a consequence of the exact controllability of the wave equation for some $T$. More recently, G. Lebeau and L. Robbiano [9] proved the null controllability without any geometric restrictions on the open subset $w$ where the control acts using Fourier 
series and sharp estimates on the eigenfunctions of the Laplacian obtained by means of Carleman's inequalities. Similar results but in a more general context including time-dependent coefficients were prove by A. Fursikov and O. Yu. Imanuvilov [7] using global Carleman's inequalities for the heat equation. In [7] local null-controllability results were also proved for semilinear heat equations (see also [8]). More recently, the connections between null and approximate controllability were investigated in [5]. In [6] the null controllability of (1.1) was proved for a class of non-linearities for which blow-up phenomena may arise.

There is a large literature on the so-called approximate controllability problem as well. System (1.1) is said to be approximately controllable in time $T$ if the reachable set $\left\{u(\cdot, T)\right.$; with $\left.h \in L^{2}\left(0, T, L^{2}(\Omega)\right)\right\}$ is dense in $L^{2}(\Omega)$ for any initial datum $u_{0} \in L^{2}(\Omega)$. In [3] the approximate controllability was proved to hold in bounded domains with globally Lipschitz non-linearities. This result was extended to the case of unbounded domains in [15]. However the properly of null-controllability is much stronger and very little is known when the domain $\Omega$ is unbounded.

Recently, in [13] the one-dimensional linear heat equation was considered in $\Omega=$ $\mathbb{R}_{+}=(0, \infty)$ with control at the extreme $x>0$. It was proved that, within the class of solutions defined by transposition, there is no smooth, compactly supported initial data that might be driven to zero in finite time.

This result shows how differently the null controllability property behaves in bounded and unbounded domains. Note that, as indicated above, approximate controllability does hold even in unbounded domains due to infinite speed of propagation. But null controllability not!

Analyzing the proof of [13] it becomes clear that such a negative result holds since we are controlling the heat equation in an unbounded domain by means of a control localized in a bounded domain.

Thus, we leave an unbounded region without control and this is the cause of the lack of null controllability. However, in this paper, even if the domain $\Omega$ is unbounded, the control acts on a large subdomain that only leaves a bounded subset uncontrolled. It is then natural to expect the positive results of the case where $\Omega$ is bounded to hold. We refer to the bibliography for a more complete list of references.

The main result of the paper is the following:

Theorem 1. Assume that $f$ is a $C^{1}$ and globally Lipschitz function, such that $f(0,0)=0$, and let $\Omega$ be an unbounded domain of class $C^{2}$ uniformly, and $w$ an 
open nonempty subset of $\Omega$ such that $\Omega \backslash w$ is bounded. Then, for all $T>0$ and for every $u_{0} \in L^{2}(\Omega)$, there exists $h \in L^{2}\left(0, T, L^{2}(\Omega)\right)$ such that the solution of $(1.1)$ satisfies (1.3). Moreover, (1.4) holds for a suitable $c>0$, independent of $u_{0}$.

In other words, system (1.1) is null controllable for all $T>0$.

Several remarks are in order:

(i) Combining the methods of this paper with those developed in [5], [6] the following additional result may be proved under the assumptions of Theorem 1.1:

- Let $v$ be any solution of system (1.1) corresponding to initial data $v_{0} \in$ $L^{2}(\Omega)$ and a control $g \in L^{2}(\Omega \times(0, T))$. Then, the solutions of (1.1) may be driven to the final state $v(T)$, i.e. for any $u_{0} \in L^{2}(\Omega)$ there exists a control such that the solution of (1.1) satisfies $u(T)=v(T)$.

- System (1.1) is approximately controllable in any time $T>0$. More precisely, for any $u_{0}, u_{1} \in L^{2}(\Omega)$ and $\varepsilon>0$ there exists a control $h \in$ $L^{2}(\Omega \times(0, T))$ such that the solution of (1.1) satisfies

$$
\left\|u(T)-u_{1}\right\|_{L^{2}(\Omega)} \leq \varepsilon .
$$

- System (1.1) is finite-approximately controllable. In other words, given any finite-dimensional subspace $E$ of $L^{2}(\Omega)$ and denoting by $\pi_{E}$ the orthogonal projection over $E$, for any $u_{0}, u_{1} \in L^{2}(\Omega)$ and $\varepsilon>0$ there exists a control $h \in L^{2}(\Omega \times(0, T))$ such that the solution of (1.1) satisfies

$$
\left\{\begin{array}{l}
\pi_{E}(u(T))=\pi_{E}\left(u_{1}\right) ; \\
\left\|u(T)-u_{1}\right\|_{L^{2}(\Omega)} \leq \varepsilon .
\end{array}\right.
$$

(ii) One may expect the same result to be true when $f$ depends both $u$ and $\nabla u$ in a globally Lipschitz way. In the case of a bounded domain this result was proved in [8]. We refer to section 4 for a more detailled discussion of this issue.

The paper is organized as follows: Section 2 is devoted to prove the null controllability of the linearized system. In Section 3 we prove Theorem 1.1 by a fixed point method. Finally in Section 4 we discuss some possible extensions of the results and methods of this paper.

Acknowledgements: This work is part of the PhD thesis of the first author developed partly in Universidad Complutense de Madrid with the support of the ALFA 
project "Amerique Latine Formation Académique" of the EU. The authors acknowledge the coordinator of this project $\mathrm{O}$. Kavian for this support.

\section{ANALYSIS OF THE LINEARIZED SYSTEM}

For the sake of completeness, first of all, we recall the definition of domain of class $C^{s}$ uniformly. We say that a domain (bounded or not) is uniformly regular of class $C^{s}(s \geq 1)$ (see [1]), if there exists an integer $r>0$ and a sequence $\left\{N_{j}\right\}$ of open subsets of $\mathbb{R}^{N}$ and homeomorphisms $\left\{\psi_{j}\right\}$ from $N_{j}$ to the unit ball in $\mathbb{R}^{N}$ such that:

i) Any $(r+1)$ distincts sets $N_{j}$ have empty intersection;

ii) $\psi_{j}\left(N_{j} \cap \Omega\right)=\left\{x:|x|<1, x_{n}>0\right\}, \quad \psi_{j}\left(N_{j} \cap \partial \Omega\right)=\left\{x:|x|<1, x_{n}=0\right\}$;

iii) If $N_{j}^{\prime}=\psi_{j}^{-1}(|x|<1 / 2), \bigcap_{j} N_{j}^{\prime}$ contains the $(1 / r)$-neighborhood of $\partial \Omega$;

iv) For $y \in N_{j}, \quad x \in \psi_{j}\left(N_{j}\right)$ we have $\left|\left(D^{\alpha} \psi_{j}\right)(y)\right| \leq r, \quad\left|\left(D^{\alpha} \psi_{j}^{-1}\right)(x)\right| \leq r$, for all $|\alpha| \leq s$.

To begin with let us consider the following initial-boundary value problem for the linear heat operator

$$
\left\{\begin{array}{l}
u_{t}-\Delta u+a u=h 1_{w} \quad \text { in } \quad \Omega \times(0, T) \\
u=0 \text { on } \Sigma=\partial \Omega \times(0, T) \\
u(x, 0)=u_{0}(x) \text { in } \Omega
\end{array}\right.
$$

where the potential $a=a(x, t)$ is assumed to be in $L^{\infty}(\Omega \times(0, T))$.

The following holds:

Theorem 2.1. Assume that the hypotheses above on $\Omega, w$ and a are satisfied. Then, for every $u_{0} \in L^{2}(\Omega)$, there exists $h \in L^{2}\left(0, T, L^{2}(\Omega)\right)$ such that the solution of $(2.1)$ satisfies (1.3). Moreover, there exists a constant $C>0$ independe of $u_{0}$ such that (1.4) holds.

Proof of Theorem 2.1. We proceed in several steps.

\section{Step 1. Observability.}

Let us consider the adjoint system 


$$
\left\{\begin{array}{l}
-\varphi_{t}-\Delta \varphi+a \varphi=0 \quad \text { in } \quad \Omega \times(0, T) \\
\varphi=0 \text { on } \quad \Sigma=\partial \Omega \times(0, T) \\
\varphi(T)=\varphi^{0} \quad \text { in } \quad \Omega
\end{array}\right.
$$

The following observability property holds:

Proposition 2.1. For all $T>0$ and $R>0$ there exists a positive constant $C$ such that

$$
|\varphi(0)|_{L^{2}(\Omega)}^{2} \leq c \int_{0}^{T} \int_{w} \varphi^{2} d x d t
$$

for every solution of (2.2) and for any $a \in L^{\infty}\left(W^{c} \times(0, T)\right)$ satisfying

$$
|a|_{L^{2}([\Omega \backslash w] \times(0, T))} \leq R
$$

for every solution of (2.2).

Remark 2.1. The constant $C$ depends on $\Omega, w$, the time $T$ and the size of the potential, but it is independent of the solution $\varphi$ of (2.2).

In order for (2.3) to be true the fact that $\Omega \mid w$ is bounded is essential. Thus, inequality (2.3) is a natural extension of the existly observability inequalities in bounded domains $([5],[7],[8], \ldots)$.

Note that in (2.3) we get an upper bound on the norm of $\varphi$ at time $t=0$, which is the final time for the adjoint system (2.2). Due to the regularizing effect of the heat equation one can not expect to get such a bound when $\|\varphi(0)\|_{L^{2}(\Omega)}$ is replaced by $\|\varphi(T)\|_{L^{2}(\Omega)}$.

Proof of Proposition 2.1: In order to prove (2.3) we introduce a "cut off" function $\rho_{1} \in C^{\infty}(\Omega)$ such that

$$
\begin{cases}\rho_{1} \geq 0 & \text { in } \quad \Omega \\ \rho_{1}=0 & \text { on } \quad w_{\varepsilon}=\{x \in w: d(x, \partial w)>\varepsilon\} \\ \rho_{1}=1 & \text { in } \quad \Omega \backslash w\end{cases}
$$

We define

$$
\theta=\rho_{1} \varphi
$$

Then $\theta$ satisfies

$$
\left\{\begin{array}{l}
-\theta_{t}-\Delta \theta=-2 \nabla \varphi \cdot \nabla \rho_{1}-\Delta \rho_{1} \varphi-a \rho_{1} \varphi \text { in } \Theta \times(0, T) \\
\theta=0 \text { on } \partial \Theta \times(0, T) \\
\partial(T)=\varphi^{0} \rho_{1} \text { in } \Theta
\end{array}\right.
$$


where

$$
\Theta=\left\{x \in \Omega: \rho_{1}(x)>0\right\} .
$$

Note that, according to the hypotheses above on $\Omega$ and $w, \Theta$ is a bounded open set. We now apply global Carleman inequalities in (2.6). Following [6] or [7], we introduce a function $\eta^{0}=\eta^{0}(x)$ such that

$$
\left\{\begin{array}{l}
\eta^{0} \in C^{2}(\bar{\Theta}) \\
\eta^{0}>0 \text { in } \Theta, \quad \eta^{0}=0 \text { on } \partial \Theta \\
\nabla \eta^{0} \neq 0 \text { in } \overline{\Theta \backslash w}
\end{array}\right.
$$

We refer to [6] for the proof of the existence of a function satisfying (2.8). Let $K_{0}>0$ be such that $K_{0} \geq 5 \max _{\Theta} \eta^{0}-6 \min _{\Theta} \eta^{0}$ and set

$$
\beta^{0}=\eta^{0}+K_{0}, \quad \bar{\beta}=\frac{5}{4} \max _{\bar{\theta}} \beta^{0}, \quad \rho^{1}(x)=e^{\lambda \bar{\beta}-\lambda \beta^{0}}
$$

where $\lambda$ is a sufficiently large positive constant that only depends on $\Theta$ and $w$ and that will be fixed later on. Notice that $\rho^{1}>0$ in $\theta$. We also introduce

$$
\phi(x, t)=\frac{\rho^{1}(x)}{[t(T-t)]}, \quad \rho(x, t)=\exp \left[\frac{\rho^{1}(x)}{[t(T-t)]}\right]=\exp (\phi(x, t))
$$

and the space

$$
Z=\left\{q \in C^{2}(\overline{\Theta \times(0, T)}): q=0 \text { on } \Sigma=\partial \Theta \times(0, T)\right\} .
$$

The following Global Carleman Inequality holds (see for instance [5]):

Proposition 2.2. There exist positive constants $C_{*}, s_{1}>0$ such that

$$
\begin{aligned}
& \frac{1}{s} \iint_{\Theta \times(0, T)} \rho^{-2 s} t(T-t)\left(\left|q_{1}\right|^{2}+|\Delta q|^{2}\right) d x d t+ \\
& +s \iint_{\Theta \times(0, T)} \rho^{-2 s} t^{-1}(T-t)^{-1}|\nabla q|^{2} d x d t+ \\
& +s^{2} \iint_{\Theta \times(0, T)} \rho^{-2 s} t^{-3}(T-t)^{-3}|q|^{2} d x d t \leq \\
& \leq C_{*}\left[\iint_{\Theta \times(0, T)} \rho^{-2 s}\left|\partial_{t} q+\Delta q\right|^{2} d x d t+\right. \\
& \left.+s^{3} \iint_{w \times(0, T)} \rho^{-2 s} t^{-3}(T-t)^{-3}|q|^{2} d x d t\right]
\end{aligned}
$$

for all $q \in Z$ and $s \geq s_{1}$. Moreover, $C_{*}$ depends only on $\Theta$ and $w$ and $s_{1}$ is of the form

$$
s_{1}=\sigma_{1}(\Theta, w)\left(T+T^{2}\right)
$$


where $\sigma_{1}(\Theta, w)$ is a positive constant that only depends on $\Theta$ and $w$.

Let us now complete the proof of the observability inequality (2.3). Let $\varphi_{0} \in L^{2}(\Omega)$ be given. By density, we can write (2.9) for $q=\theta$, with $\theta$ being the solution of the system (2.6). Taking into account that $\theta_{t}+\Delta \theta=2 \nabla \varphi \cdot \nabla \rho_{1}+\Delta \rho_{1} \varphi+a \rho_{1} \varphi$, it follows that

$$
\begin{aligned}
& s \cdot \iint_{\Theta \times(0, T)} \rho^{-2 s} t^{-1}(T-t)^{1} d x d t+ \\
& s^{3} \iint_{\Theta \times(0, T)} \rho^{-2 s} t^{-3}(T-t)^{-3}|\theta|^{2} d x d t \leq \\
& \leq c_{*}\left[\iint_{\Theta \times(0, T)} \rho^{-2 s}\left|2 \nabla \varphi \cdot \nabla \rho_{1}+\Delta \rho_{1} \varphi+a \rho_{1} \varphi\right|^{2} d x d t+\right. \\
& \left.+s^{3} \iint_{w \times(0, T)} \rho^{-2 s} \cdot t^{-3}|\theta|^{2} d x d t\right] \leq \\
& \leq c_{*}\left[4 \iint_{\Theta \times(0, T)}\left|\nabla \varphi \cdot \nabla \rho_{1}\right|^{2} \rho^{-2 s} d x d t+4 \iint_{\Theta \times(0, T)}^{*}\left|\nabla \varphi \cdot \nabla \rho_{1} \Delta \rho_{1} \varphi\right| d x d t+\right. \\
& +4 \iint_{\Theta \times(0, T)} \rho^{-2 s}\left|\nabla \varphi \cdot \nabla \rho_{1} a \rho_{1} \varphi\right| d x d t+\iint_{\Theta \times(0, T)} \rho^{-2 s}\left|\Delta \rho_{1} \varphi\right|^{2} d x d t+ \\
& +2 \iint_{\Theta \times(0, T)} \rho^{-2 s}\left|\Delta \rho_{1} \varphi a \rho_{1}\right| d x d t+\iint_{\Theta \times(0, T)} \rho^{-2 s}\left|a \rho_{1} \varphi\right|^{2} d x d t+ \\
& \left.+s^{3} \iint_{w \times(0, T)} \rho^{-2 s} t^{-3}(T-t)^{-3}\left|\rho_{1} \varphi\right|^{2} d x d t\right] \leq \\
& \leq c_{*}\left[c_{0} T^{6} \iint_{w \times(0, T)} \rho^{-2 s} t^{-3}(T-T)^{-3}|\nabla \varphi|^{2} d x d t+\right. \\
& +c_{0} T^{6} \iint_{w \times(0, T)} \rho^{-2 s} t^{-3}(T-t)^{-3}|\varphi|^{2} d x d t+ \\
& +c_{0} T^{6} \iint_{\Theta \times(0, T)} \rho^{-2 s} t^{-3}(T-t)^{-3}|\theta|^{2} d x d t+ \\
& \left.+c_{0} s^{3} \iint_{w \times(0, T)} \rho^{-2 s} t^{-3}(T-t)^{-3}|\varphi|^{2} d x d t\right]
\end{aligned}
$$

where $c_{0}$ depends on the norm of $\rho_{1}$ in $L^{\infty}\left(0, T, W^{2, \infty}(\Omega)\right)$ and $|a|_{L^{\infty}([\Omega \backslash w] \times(0, T))}$. Let $s \geq s_{2}=\max \left\{s_{1}, c_{0}^{1 / 3} T^{2}\right\}$. We have 


$$
\begin{aligned}
& \iint_{\Theta \times(0, T)} \rho^{2 s} t^{-1}(T-t)^{-1}|\nabla \theta|^{2} d x d t \leq \\
& \leq c_{*}\left(c_{0}+1\right) s^{2}\left[\iint_{w \times(0, T)} \rho^{-2 s} t^{-3}(T-t)^{-3}|\nabla \varphi|^{2} d x d t+\right. \\
& \left.+\iint_{w \times(0, T)} \rho^{2 s} t^{-3}(T-t)^{-3}|\varphi|^{2} d x d t\right]
\end{aligned}
$$

for all $s \geq s_{2}$.

Let us estimate the weights appearing in (2.12):

Lemma 2.1. One has

$$
\left|\rho^{-2 s} t^{-3}(T-t)^{-3}\right|_{\infty} \leq 2^{6} T^{-6} \exp \left(-c_{s} T^{-2}\right)
$$

for all

$$
s \geq s_{3}=\max \left\{s_{2}, 3 T^{2}\left(8 \min _{\bar{\theta}} \rho^{1}(x)\right)^{-1}\right\}
$$

Proof of Lemma 2.1: We observe that

$$
\rho(x, t)^{-2 s} t^{-3}(T-t)^{-3}=\frac{1}{f_{x}(t)}
$$

for any $x \in \Theta, t \in(0, T)$ with

$$
f_{x}(t)=t^{3}(T-t)^{-3} \exp \left(\frac{2 s \rho^{1}(x)}{t(T-t)}\right)=\tau^{3} \exp \left(\frac{2 s \rho^{1}(x)}{\tau}\right)=g_{x}(\tau)
$$

and $\tau=t(T-t) \in\left[0, T^{2} / 4\right]$.

The minimum of $g_{x}$ is achieved at $\hat{\tau}=\frac{2}{3} s \rho^{1}(x)$ and $g_{x}(\hat{\tau})=\left(\frac{2}{3} s \rho^{1}(x)\right)^{3} e^{3}$. On the other hand, $g_{x}(0)=\infty$ and $g_{x}$ is decreasing for $\tau \in(0, \hat{\tau})$. and increasing for $\tau>\hat{\tau}$. Thus,

$$
\begin{gathered}
\min _{0 \leq t \leq T} f_{x}(t)=\min _{0 \leq \tau \leq T^{2} / 4} g_{x}(\tau)= \\
=\left\{\begin{array}{l}
g_{x}(\hat{\tau})=\left(\frac{2}{3} s \rho^{1}(x)\right)^{3} e^{3} \text { if } T^{2} / 4 \geq \frac{2}{3} s \rho^{1}(x) \\
g_{x}\left(T^{2} / 4\right)=2^{-6} T^{6} \exp \left(8 s \rho^{1}(x) T^{-2}\right) \text { if } \frac{T^{2}}{4}<\frac{2}{3} s \rho^{1}(x) .
\end{array}\right.
\end{gathered}
$$

Therefore, if $s \geq s_{3}$ as in (2.13), we have

$$
\min _{0 \leq t \leq T} f_{x}(t) \geq 2^{-6} T^{6} \exp \left(c s T^{-2}\right) \text { with } c=8 \min _{\bar{\theta}} \rho^{1}(x) .
$$


In view of (2.14) and (2.16), (2.13) holds.

Remark 2.2. In view of (2.10) and (2.13), we have

$$
s_{3} \leq s_{4}=c\left(T+\left(1+c_{0}\right) T^{2}\right) .
$$

Applying (2.13) with $s$ as in the right hand side of (2.17), we deduce that

$$
\left|\rho^{-2 s} t^{-3}(T-t)^{-3}\right|_{-} \infty \leq 2^{6} T^{-6} \exp \left(-c\left(1+c_{0}+\frac{1}{T}\right)\right) .
$$

Lemma 2.2. One has

$$
\rho^{-2 s} t^{-1}(T-t)^{-1} \geq \frac{16}{3} T^{-2} \exp \left(-c s T^{-2}\right)
$$

for all $x \in \Theta$ and $t \in[T / 4,3 T / 4]$, whenever $s \geq s_{4}$.

Proof of Lemma 2.2: We have

$$
\rho(x, t)^{-2 s} t^{-1}(T-t)^{-1}=\frac{1}{h_{x}(t)}
$$

with

$$
h_{x}(t)=t(T-t) \exp \left(\frac{2 s \rho^{1}(x)}{t(T-t)}\right)=\tau \exp \left(\frac{2 s \rho^{1}(x)}{\tau}\right)=j_{x}(\tau)
$$

and $\tau=t(T-t) \in\left[0, T^{2} / 4\right]$.

When $t \in[T / 4,3 T / 4]$, one has $\tau \in\left[\frac{3 T^{2}}{16}, \frac{T^{2}}{4}\right]$.

Proceeding as in the proof of Lemma 2.1, we deduce that

$$
\max _{T / 4 \leq t \leq 3 T / 4} h_{x}(t) \leq \frac{3}{16} T^{2} \exp \left(c s T^{-2}\right),
$$

provided $s \geq T^{2}\left(8 \min _{\bar{\theta}} \rho^{1}(x)\right)^{-1}$. In particular, this is the case if $s \geq s_{4}$. Therefore

$$
\rho^{-2 s} t^{-1}(T-t)^{-1} \geq \frac{16}{3} T^{-2} \exp \left(-c s T^{-2}\right)
$$

in $\Theta \times[T / 4,3 T / 4]$ whenever $s \geq s_{4}$. This concludes the proof.

Remark 2.3. Arguing as in Remark 2.2, it follows that

$$
\rho^{-2 s} t^{-1}(T-t)^{-1} \geq \frac{16}{3} T^{-2} \exp \left(-c\left(1+\frac{1}{T}+c_{0}\right)\right) \text { in } \Theta \times[T / 4,3 T / 4]
$$
for $s=s_{4}$.

Coming back to (2.12) and using (2.18) and (2.20) we deduce 


$$
\iint_{\Theta \times(T / 4,3 T / 4)}|\nabla \theta|^{2} d x d t \leq c_{1}\left[\iint_{w \times(0, T)}\left(\varphi^{2}+|\nabla \varphi|^{2}\right) d x d t\right]
$$

where $c_{1}=\exp \left(\tilde{c}\left(1+c_{0}+\frac{1}{T}\right)\right)$.

Let $\lambda_{1}$ be the first eigenvalue of $-\Delta$ in $H_{0}^{1}(\theta)$. In view of $(2.21), \theta=\rho_{1} \varphi$ and also according to the choice of $\rho_{1}$, we deduce that

$$
\iint_{\Omega \times(T / 4,3 T / 4)} \varphi^{2} d x d t \leq c_{2}\left[\iint\left(\varphi^{2}+|\nabla \varphi|^{2}\right) d x d t\right] .
$$

Multiplying in (2.22) by $\varphi$ and integrating in $\Omega$, we deduce that

$$
-\frac{1}{2} \frac{d}{d t}|\varphi|^{2}+\int_{\Omega}|\nabla \varphi|^{2} d x+\int_{\Omega} a \varphi^{2} d x=0 .
$$

Integrating this equality in $[0, t]$ for any $t \in[0, T]$, we find

$$
\int_{\Omega} \varphi(0)^{2} d x \leq c_{3} \int_{\Omega} \varphi^{2}(t) d x . \quad \forall t \in[0, T] .
$$

It follows that

$$
\int_{\Omega} \varphi(0)^{2} d x \leq c_{3} \frac{2}{T} \iint_{\Omega \times(T / 4,3 T / 4)} \varphi^{2} d x .
$$

In view of (2.25) and (2.22), we deduce that

$$
|\varphi(0)|_{L^{2}(\Omega)}^{2}=c_{4}\left[\iint_{w \times(0, T)}\left(\varphi^{2}+|\nabla \varphi|^{2}\right) d x d t\right] .
$$

Let $K$ an compact subset of $\theta$ and $\delta>0$. Proceeding as in the proof of (2.26), we have

$$
|\varphi(0)|_{L^{2}(\Omega)}^{2} \leq c_{4}\left[\iint_{(\delta, T-\delta) \times K}\left(\varphi^{2}+|\nabla \varphi|^{2}\right) d x d t\right]
$$

By using regularity theory for the heat equation one obtains, from (2.27), that .

$$
|\varphi(0)|_{L^{2}(\Omega)}^{2} \leq c \int_{0}^{T} \int_{w} \varphi^{2} d x d t
$$

which is the observability inequality (2.3).

In fact, one has the following result:

Lemma 2.3. Let $A$ be a bounded open regular subset of $\mathbb{R}^{N}$. If $\psi \in L^{2}\left(0, T, L^{2}(A)\right)$ is a solution of

$$
\left\{\begin{array}{l}
\psi_{t}-\Delta \psi+a \psi=0 \text { in } A \times(0, T) \\
\psi=0 \text { on } \partial A \times(0, T) \\
\psi(x, 0)=\psi^{0}(x) \text { in } A
\end{array}\right.
$$


then $\nabla \psi \in L_{\text {loc }}^{2}(A \times(0, T))$.

Proof of Lemma 2.3: Given $\delta>0$ and a compact set $K \subset A$ let us define $\xi=\psi(x, t) \varepsilon(t) \eta(x)$, where

- $\varepsilon$ is a regular function, $\varepsilon=1$ in $(\delta, T-\delta)$ and $\varepsilon(0)=\varepsilon(T)=0$.

- $\eta$ is a regular function, $\eta=0$ on the boundary $\partial A$ of $A, \quad \eta=1$ in $K$.

Then $\xi$ satisfies

$$
\left\{\begin{array}{l}
\xi_{t}-\Delta \xi+a \xi=\varepsilon_{t} \psi \eta-2 \varepsilon \nabla \psi \cdot \nabla \eta-\varepsilon \psi \Delta \eta \text { in } A \times(0, T) \\
\xi=0 \text { on } \partial A \times(0, T) \\
\xi(0)=0
\end{array}\right.
$$

Thus, by using energy estimates, one has

$$
|\nabla \xi|_{L^{2}(A \times(0, T))} \leq c\left|\varepsilon_{t} \psi \eta-2 \varepsilon \nabla \psi \cdot \nabla \eta-\varepsilon \psi \Delta \eta\right|_{L^{2}\left(0, T, H^{-1}(A)\right)}
$$

where $c=c(a)$.

Furthermore, we remark that

$$
\left|\varepsilon_{t} \psi \eta-2 \varepsilon \nabla \psi \cdot \nabla \eta-\varepsilon \psi \Delta \eta\right|_{-} L^{2}\left(0, T, H^{-1}(A)\right) \dot{\dot{x}} c|\psi|_{L^{2}\left(0, T, L^{2}(A)\right)}
$$

and in view of $\nabla \xi=\nabla \psi$ in $K \times(\delta, T-\delta)$ we get

$$
|\nabla \psi|_{L^{2}\left(\delta, T-\delta, L^{2}(K)\right)} \leq c|\psi|_{L^{2}\left(0, T, L^{2}(A)\right)}
$$

In view this, we deduce that

$$
\int_{(\delta, T-\delta) \times K}|\nabla \varphi|^{2} d x d t \leq c \int_{0}^{T} \int_{w} \varphi^{2} d x d t .
$$

Thus, (2.28) holds.

\section{Step 2. Approximate controllability.}

In view of the uniform observability inequality (2.3) the null controllability result of Theorem 1.1 can be proved as the limit of an approximate controllability property.

Let us first discuss the approximate controllability.

Given $u_{0} \in L^{2}(\Omega)$ and $\delta>0$ we introduce the quadratic functional

$$
J_{\delta}\left(\varphi^{0}\right)=\frac{1}{2} \int_{0}^{T} \int_{\omega} \varphi^{2} d x d t+\delta\left|\varphi^{0}\right|_{L^{2}(\Omega)}+\int_{\Omega} u_{0} \varphi(0) d x .
$$

where $\varphi$ denotes the solution of $(2.2)$ with initial data $\varphi^{0}$. The functional $J_{\delta}$ is continuous and convex in $L^{2}(\Omega)$. Moreover, $J_{\delta}$ is coercive. More precisely, in view of (2.3), one has

$$
\liminf _{\left|\varphi^{0}\right|_{L^{2}(\Omega)} \rightarrow \infty} \frac{J_{\delta}\left(\varphi^{0}\right)}{\left|\varphi^{0}\right|_{L^{2}(\Omega)}} \geq \delta .
$$


Then $J_{\delta}$ has a unique minimizer in $L^{2}(\Omega)$. Let us denote it by $\hat{\varphi}^{0, \delta}$. It is easy see that the control $h_{\delta}=\hat{\varphi}^{\delta}$, where $\hat{\varphi}^{\delta}$ is the solution of (2.2) associated to the minimizer $\hat{\varphi}^{0, \delta}$, is such that the solution of $(2.1)$ satisfies of $J_{\delta}$

$$
\left|u_{\delta}(T)\right|_{L^{2}(\Omega)} \leq \delta .
$$

We refer to [3] for the details of the proof. In order to get a uniform bound on the control we observe that, by (2.3),

$$
\begin{gathered}
J_{\delta}\left(\varphi^{0}\right) \geq \frac{1}{2} \int_{0}^{T} \int_{\omega} \varphi^{2} d x d t- \\
-c\left[\int_{0}^{T} \int_{\omega} \varphi^{2} d x d t\right]^{1 / 2}\left|u_{0}\right|_{L^{2}(\Omega)}
\end{gathered}
$$

with $c>0$ independent of $\delta$. On the other hand,

$$
J_{\delta}\left(\hat{\varphi}^{0, \delta}\right) \leq J_{\delta}(0)=0 .
$$

Combining (2.34) and (2.35) we deduce that

$$
\left|h_{\delta}\right|_{L^{2}\left(0, T, L^{2}(\Omega)\right)} \leq \frac{c}{2}\left|u_{0}\right|_{L^{2}(\Omega)}, \text { for all } \delta>0 .
$$

In other words, $h_{\delta}$ remains bounded in $L^{2}\left(0, T, L^{2}(\Omega)\right)$ as $\delta \rightarrow 0$.

\section{Step 3. Null controllability.}

Extracting subsequences we deduce that

$$
h_{\delta} \rightarrow h \quad \text { as } \delta \rightarrow 0 \text { weakly in } L^{2}(\Omega \times(0, T)),
$$

for some $h \in L^{2}(\Omega \times(0, T))$.

It is easy to see that the limit $h$ is such that the solution $u$ of (2.1) satisfies (1.3). Moreover, by lower semicontinuity of the norm with respect to the weak topology and in view of (2.37) we deduce that:

$$
|h|_{L^{2}(\Omega \times(0, T))} \leq \liminf _{\delta \rightarrow 0}\left|h_{\delta}\right|_{L^{2}(\Omega \times(0, T))} \leq \frac{c}{2}\left|u_{0}\right|_{L^{2}(\Omega)} .
$$

This concludes the proof of Theorem 2.1.

\section{Proof of the main result}

We are now ready to prove Theorem 1.1.

Proof of Theorem 1.1: First of all note that system (1.1) can be written as follows:

$$
\left\{\begin{array}{l}
u_{t}-\Delta u+\left(1-1_{w}\right) f(u)=h 1_{w}-f(u) 1_{w} \text { in } \Omega \times(0, T) \\
u=0 \text { on } \partial \Omega \times(0, T) \\
u(x, 0)=u_{0}(x) \text { in } \Omega
\end{array}\right.
$$


Therefore it is sufficient to analyze the controllability of the system:

$$
\left\{\begin{array}{l}
u_{t}-\Delta u+\left(1-1_{w}\right) f(u)=q(x, t) 1_{w} \text { in } \Omega \times(0, T) \\
u=0 \text { on } \partial \Omega \times(0, T) \\
u(x, 0)=u_{0}(x) \text { in } \Omega
\end{array}\right.
$$

Indeed, if $q$ is the control for (3.2), $h=f(u)+q$ is the control for (1.1) and viceversa. The advantage of writing system (1.1) in the form (3.2) is that the nonlinearity is now localized in a bounded subdomain of $\Omega$, according to property (1.2), and this is important to guarantee the compactness properties that are needed to apply the fixed point argument.

We introduce the non-linearity

$$
g(s)= \begin{cases}\frac{f(s)}{s}, & \text { if } \quad s \neq 0 \\ f^{\prime}(0), & \text { if } \quad s=0 .\end{cases}
$$

Note that $g$ is a uniformly bounded function with $|g|_{\infty} \leq\left|f^{\prime}\right|_{\infty}$.

Given any $v \in L^{2}([\Omega \backslash w] \times(0, T))$ we consider the "linearized" system:

$$
\left\{\begin{array}{l}
u_{t}-\Delta u+1_{[\Omega \backslash w]} g(v)=q 1_{w} \text { in } \Omega \times(0, T) \\
u=0 \text { on } \partial \Omega \times(0, T) \\
u(x, 0)=u_{0}(x) \text { in } \Omega
\end{array}\right.
$$

where $\Omega \mid w$ denotes the complement of $w$ in $\Omega$ and $1_{[\Omega \mid w]}$ its characteristic function.

Observe that (3.4) is a linear system on the state $u$ with potential $a=g(v) \in$ $L^{\infty}([\Omega \backslash w] \times(0, T))$ satisfying the following bound

$$
|a|_{L^{\infty}([\Omega \backslash w] \times(0, T))} \leq\left|f^{\prime}\right|_{L^{\infty}(\mathbb{R})} .
$$

With this notation system (3.4) may be rewritten in the form

$$
\left\{\begin{array}{l}
u_{t}-\Delta u+1_{[\Omega \backslash w]} a u=q 1_{w} \text { in } \Omega \times(0, T) \\
u=0 \text { on } \partial \Omega \times(0, T) \\
u(x, 0)=u_{0}(x) \text { in } \Omega
\end{array}\right.
$$

As we saw in section $2, s>0$ being fixed, for any $v \in L^{2}\left(0, T, L^{2}(\Omega \backslash w)\right)$ this allows us to define a control $q_{\delta}=q_{\delta}(x, t, v) \in L^{2}\left(0, T, L^{2}(\Omega)\right)$ such that the solution $u_{\delta}$ of (3.6) satisfies

$$
\left|u_{\delta}(T)\right|_{L^{2}(\Omega)} \leq \delta
$$

Moreover, for every $R>0$ and potential satisfying $|a|_{L^{\infty}([\Omega \backslash w] \times(0, T))} \leq R$, we have:

$$
\left|q_{\delta}\right|_{L^{2}\left(0, T, L^{2}(\Omega)\right)} \leq c\left|u_{0}\right|_{L^{2}(\Omega)} .
$$

Therefore, the controls $q_{\delta}$ are uniformly bounded in $L^{2}(w \times(0, T))$. 
This allows to build a nonlinear mapping

$$
N: L^{2}\left(0, T, L^{2}(\Omega \backslash w)\right) \rightarrow L^{2}\left(0, T, L^{2}(\Omega \backslash w)\right), \quad N(v)=u .
$$

Thus, the approximate control problem for system (3.2) is reduced to find a fixed point for the map $N$. Indeed, if $v \in L^{2}\left(0, T, L^{2}\left(w^{c}\right)\right)$ is such that $N(v)=u, u$ solution of (3.6) is actually solution of (3.2). Then, the control $q_{\delta}=q_{\delta}(v)$ is the one we were looking for since, by construction, $u_{\delta}=u_{\delta}(v)$ satisfies (3.7).

As we shall see, the nonlinear map $N$ satisfies the following two properties:

$N$ is continuous and compact

$$
\left\{\begin{array}{l}
\text { the range of } N \text { is bounded, i.e., } \exists M>0 \text { : } \\
|N(v)|_{L^{2}\left(0, T, L^{2}(\Omega \backslash w)\right)} \leq M, \quad \forall v \in L^{2}\left(0, T, L^{2}(\Omega \backslash w)\right)
\end{array}\right.
$$

In view of these two properties and as a consequence of Schauder's fixed point Theorem, the existence of a fixed point of $N$ follows immediately. We shall return later to the proof of (3.9) and (3.10). By the moment let us assume that these properties hold. Then, we have found a control $q_{\delta}$ in $L^{2}\left(0, T, L^{2}(w)\right)$ such that the solution $u_{\delta}$ of

$$
\left\{\begin{array}{l}
u_{\delta}^{\prime}-\Delta u_{\delta}+1_{[\Omega \backslash w]} f\left(u_{\delta}\right)=q_{\delta}(x, t) 1_{w} \text { in } \Omega \times(0, T) \\
u_{\delta}=0 \text { on } \partial \Omega \times(0, T) \\
u_{\delta}(x, 0)=u_{0}(x) \text { on } \Omega
\end{array}\right.
$$

satisfies

$$
u_{\delta}(T)=0 \quad \text { in } \Omega
$$

with an estimate of the form

$$
\left|q_{\delta}\right|_{L^{2}\left(0, T, L^{2}(\Omega)\right)} \leq c\left|u_{0}\right|_{L^{2}(\Omega)} .
$$

Passing to the limit as $\delta \rightarrow 0$, as in section 2, we deduce the existence of a limit control $q \in L^{2}\left(0, T, L^{2}(\Omega)\right)$ such that the solution $u$ of (3.2) satisfies (1.3) and (1.4). Let us now return to the proof of (3.9) and (3.10).

Continuit of $N$. Assume that $v_{j} \rightarrow v$ in $L^{2}\left(0, T, L^{2}(\Omega \backslash w)\right)$. Then the potential $a_{j}=g\left(v_{j}\right)$ is such that

$$
a_{j}=g\left(v_{j}\right) \rightarrow a=g(v) \quad \text { in } \quad L^{p}([\Omega \backslash w] \times(0, T))
$$

for all $1 \leq p<\infty$ and

$$
\left|a_{j}\right|_{L^{\infty}([\Omega \backslash w] \times(0, T))} \leq L,
$$

with $L$ the Lipschitz constant of $f$. According to Proposition 3.1 the corresponding controls are uniformly bounded:

$$
\left|q_{j}\right|_{L^{2}\left(0, T, L^{2}(\Omega)\right)} \leq c, \quad \forall j \geq 1
$$


and, more precisely,

$$
q_{j}=\hat{\varphi}_{j} \quad \text { in } \quad w \times(0, T)
$$

where $\hat{\varphi}_{j}$ solves

$$
\left\{\begin{array}{l}
-\varphi_{t}-\Delta \varphi+1_{[\Omega \backslash w]} g\left(v_{j}\right) \varphi=0 \text { in } \Omega \times(0, T) \\
\varphi=0 \text { on } \partial \Omega \times(0, T) \\
\varphi(T)=\hat{\varphi}_{j}^{0} \text { in } \Omega
\end{array}\right.
$$

with the datum $\hat{\varphi}_{j}^{0}$ minimizing the corresponding functional in $L^{2}(\Omega)$. We also have

$$
\left|\hat{\varphi}_{j}^{0}\right|_{L^{2}(\dot{\Omega})} \leq c .
$$

By extracting subsequences we have

$$
\hat{\varphi}_{j}^{0} \rightarrow \hat{\varphi}^{0} \quad \text { weakly in } \quad L^{2}(\Omega)
$$

and in view of (3.14)-(3.15), we deduce that

$$
\hat{\varphi}_{j} \rightarrow \hat{\varphi} \quad \text { weakly in } L^{2}\left(0, T, H_{0}^{1}(\Omega)\right)
$$

where $\hat{\varphi}$ solves

$$
\left\{\begin{array}{l}
-\varphi_{t}-\Delta \varphi+1_{[\Omega \backslash w]} g(v) \varphi=0 \text { in } \Omega \times(0, T) \\
\varphi=0 \text { on } \partial \Omega \times(0, T) \\
\varphi(T)=\varphi^{0} \text { in } \Omega
\end{array}\right.
$$

We also have that

$$
\partial_{t} \hat{\varphi}_{j} \quad \text { is bounded in } \quad L^{2}\left(0, T, H^{-1}(\Omega)\right),
$$

and, once again, by Aubin-Lions compactness lemma, it follows that

$$
\hat{\varphi}_{j} \rightarrow \hat{\varphi} \quad \text { strongly in } L^{2}\left(0, T, L^{2}(\Omega \backslash w)\right) .
$$

\section{Consequently}

$$
q_{j} \rightarrow q \text { in } L^{2}\left(0, T, L^{2}(\Omega \backslash w)\right)
$$

where

$$
q=\hat{\varphi} \quad \text { in } \quad w \times(0, T) .
$$

It is then easy to see that

$$
u_{j} \rightarrow u \quad \text { in } \quad L^{2}\left(0, T, L^{2}(\Omega \backslash w)\right),
$$

where

$$
\left\{\begin{array}{l}
u_{t}-\Delta u+1_{[\Omega \backslash w]} g(v) u=q 1_{w} \text { in } \Omega \times(0, T) \\
u=0 \text { on } \partial \Omega \times(0, T) \\
u(x, 0)=u_{0}(x) \text { in } \Omega
\end{array}\right.
$$

and 


$$
|u(T)|_{L^{2}(\Omega)} \leq \delta
$$

To conclude the continuity of $N$ it is sufficient to check that the limit $\hat{\varphi}^{0}$ in (3.20) is the minimizer of the function $J$ associated to the limit control problem (3.28)-(3.29). To do this, given $\psi^{0} \in L^{2}(\Omega)$ we have to show that

$$
J\left(\hat{\varphi}^{0}\right) \leq J\left(\psi^{0}\right) .
$$

But this is immediate since, by lower semicontinuity, we have

$$
J\left(\hat{\varphi}^{0}\right) \leq \liminf _{j \rightarrow \infty} J_{i}\left(\hat{\varphi}_{j}^{0}\right),
$$

on one hand,

$$
J\left(\psi^{0}\right)=\liminf _{j \rightarrow \infty} J_{j}\left(\psi^{0}\right), \quad \forall \psi^{0} \in L^{2}(\Omega)
$$

on the other one, and finally

$$
J_{j}\left(\hat{\varphi}_{j}^{0}\right) \leq J_{j}\left(\psi^{0}\right), \quad \forall \varphi^{0} \in L^{2}(\Omega), \quad j \geq 1
$$

since $\hat{\varphi}_{j}^{0}$ is the minimizer of $J_{j}$.

Compactness of $N$. The arguments above show that when $v$ lies in a bounded set $B$ of $L^{2}([\Omega \mid w] \times(0, T)), \quad u=N(v)$ also lies in an bounded set of $L^{2}([\Omega \mid w] \times(0, T))$. We have to show that $N(B)$ is relatively compact in $L^{2}([\Omega \mid w] \times(0, T))$. But this can be obtained easily by means of the regularizing effect of the heat equation. Indeed, we have

$$
\left\{\begin{array}{l}
u_{t}-\Delta u=\beta \text { in } \Omega \times(0, T) \\
u=0 \text { on } \partial \Omega \times(0, T) \\
u(x, 0)=u_{0}(x) \text { in } \Omega
\end{array}\right.
$$

with $\beta=q 1_{w}-1_{[\Omega \mid w]} g(v) u$ which is uniformly bounded in $L^{2}\left(0, T, L^{2}(\Omega)\right)$. Then, by classical regularity results on the heat equation we deduce that $u$ is uniformly bounded in $L^{2}\left(0, T, H_{0}^{1}(\Omega)\right) \cap H^{1}\left(0, T, H^{-1}(\Omega)\right)$. Therefore, as a consequence of Aubin-Lions compactness lemma, $u$ lies in a relatively compact set of $L^{2}\left(0, T, L^{2}(\Omega \backslash w)\right)$.

Boundedness of the range of $\boldsymbol{N}$. According (3.13), there exists $c>0$ such that the control $q=q(v)$ satisfies

$$
|q(v)|_{L^{2}\left(0, T, L^{2}(\Omega \backslash w)\right)} \leq c .
$$

Classical energy estimates for the system (3.28) show that

$$
|u(v)|_{L^{2}\left(0, T, L^{2}(\Omega \backslash w)\right)} \leq c
$$

as well, since the potential involved in it is uniformly bounded. 
This concludes the proof of Theorem 1.1.

\section{Further comments}

The results of this paper can be extended to more general equations of the form

$$
\left\{\begin{array}{l}
u_{t}-\Delta u+a u+\operatorname{div}(B u)=h 1_{w} \text { in } \Omega \times(0, T) \\
u=0 \text { on } \partial \Omega \times(0, T) \\
u(x, 0)=u_{0}(x) \text { in } \Omega
\end{array}\right.
$$

where $a \in L^{\infty}(\Omega \times(0, T))$ and $B \in\left(L^{\infty}(\Omega \times(0, T))\right)^{N}$. To do that, it is sufficient to obtain appropriate observability for the adjoint system

$$
\left\{\begin{array}{l}
-\varphi_{t}-\Delta \varphi+a \varphi-B \cdot \nabla \varphi=0 \text { in } \Omega \times(0, T) \\
\varphi=0 \text { on } \partial \Omega \times(0, T)
\end{array}\right.
$$

This can be done following the methods developed in this paper with minor changes. More precisely, arguing as in section 3, we find that

$$
|\varphi(0)|_{L^{2}(\Omega)}^{2} \leq c \int_{0}^{T} \int_{w} \varphi^{2} d x d t
$$

for any solution of (4.2) and for all $a \in L^{\infty}(w \times(0, T)), \quad B \in\left(L^{\infty}(w \times(0, T))^{N}\right.$.

Moreover, the observability constant $c$ in (4.3) remains bounded when the potentials $a$ and $B$ lie in a bounded set of $L^{\infty}(\Omega \times(0, T))$ and $\left(L^{\infty}(\Omega \times(0, T))\right)^{N}$ respectively.

The situation is different when the state equation is of the form

$$
\left\{\begin{array}{l}
u_{t}-\Delta u+B \cdot \nabla u=0 \text { in } \Omega \times(0, T) \\
u=0 \text { on } \partial \Omega \times(0, T) \\
u(x, 0)=u_{0}(x) \text { in } \Omega
\end{array}\right.
$$

Obviously, when $B \in\left(W^{1, \infty}(\Omega \times(0, T))\right)^{N}$, system (4.4) can be written in the form (4.1) and the methods of this paper apply. However, when $B$ is only assumed to be in $\left(L^{\infty}(\Omega \times(0, T))\right)^{N}$, the situation is much more delicate. Indeed, the adjoint system takes the form

$$
\left\{\begin{array}{l}
-\varphi_{t}-\Delta \varphi-\operatorname{div}(B \varphi)=0 \text { in } \Omega \times(0, T) \\
\varphi=0 \text { on } \partial \Omega \times(0, T)
\end{array}\right.
$$

and, therefore, the global Carleman inequality of Proposition 2.2 cannot be applied without further regularity assumptions on $B$. Roughly speaking, in order to address system (4.5) we would need the $H^{-1}$-version of the $L^{2}$-Carleman inequality in Proposition 2.2. This problem was recently solve by O. Yu. Imanuvilov and M. Yamamoto [IY] in the case of a bounded domain. They proved the Carleman inequality for the system (4.5), only supposing $B \in\left(L^{\infty}(\Omega \times(0, T))\right)^{N}$. Then by a fixed point method they proved null controllability for the semilinear heat equation 


$$
\left\{\begin{array}{l}
u_{t}-\Delta u+f(x, t, u, \nabla u)=h 1_{w} \text { in } \Omega \times(0, T) \\
u=0 \text { on } \partial \Omega \times(0, T) \\
u(x, 0)=u_{0}(x) \text { in } \Omega
\end{array}\right.
$$

when $\Omega$ is an bounded set of $\mathbb{R}^{N}$, and $f$ is globally Lipschitz.

The extension of this result to the case where $\Omega$ is unbounded, $\Omega \backslash w$ being bounded, is an open problem.

\section{BIBLIOGRAFíA}

[1] F.E. Browder, Estimates and existence theorems for elliptic boundary value problems, Proc. N.A.S. $45,365-375$ (1959).

[2] C. Fabre, Uniqueness results for Stokes equations and their consequences in linear and nonlinear control problems, ESAIM: COCV 1 (1996), pp. 267-302 (Web site http://www.emath.fr/cocv/).

[3] C. Fabre, J.P. Puel and E. Zuazua, Approximate controllability of the semilinear heat equation, Proc. Royal Soc. Edinburgh, 125A (1995), 31-61.

[4] E. Fernández-Cara, Null controllability of the semilinear heat equation, ESAIM: COCV, 2 (1997), 87-107.

[5] E. Fernández-Cara and E. Zuazua, The cost of approximate controllability for heat equations: the linear case, Advances Diff. Equations, to appear.

[6] E. Fernández-Cara and E. Zuazua, Controllability of weakly blowing-up semilinear heat equations, Annales IHP, Analyse non-linéaire, to appear.

[7] A.V. Fursikov and O. Yu. Imanuvilov, Controllability of evolution equations, Lectures notes series 34, SNU, Seoul, 1996.

[8] O. Yu. Imanuvilov and M. Yamamoto, On Carleman inequalities for parabolic equations in Sobolev spaces of negative order and exact controllability for semilinear parabolic equations, preprint \# 98-46, University of Tokyo. Graduate School of Mathematics, Japan (1998).

[9] G. Lebeau and L. Robbiano, Contrôle exacte de l'équation de la chaleur, Comm. P.D.E., 20 (1995), 335-356.

[10] J.L. Lions, Remarks on approximate controllability, J. Anal. Math., 59 (1993), 103-116.

[11] J.L. Lions, Remarques sur la contrôllabilité approchée, in "Jornadas Hispano-Francesas sobre control de Sistemas Distribuidos", University of Málaga, Spain (1991), 77-87.

[12] J.L. Lions and E. Zuazua, The cost of controlling unstable systems: time irreversible system, Rev. Mat. Complutense, 10 (2) (1997), pp. 481-523.

[13] S. Micu and E. Zuazua, On the lack of null controllability of the heat equation on the half line, Trans. A.M.S., to appear.

[14] D.L. Russell, A unified boundary controllability theory for hyperbolic and parabolic partial differential equations, Studies in Appl. Math., 52 (1973), 189-221.

[15] L. de Teresa and E. Zuazua, Approximate controllability of the semilinear heat equation in unbounded domains, Nonlinear Anal. T.M.A., 37 (8) (1999), 1059-1090.

[16] E. Zuazua, Finite Dimensional Null Controllability for the Semilinear Heat Equation, J. Math. Pures Appl., 76 (1997), 237-264.

[17] E. Zuazua, Approximate controllability of the semilinear heat equation: boundary control, in Computational Sciences for the 21st Century, M.O. Bristeau et al. eds. John Wiley \& Sons, (1997), pp. 738-747.

[18] E. Zuazua, Approximate Controllability for Semilinear Heat Equation with Globally Lipschitz Nonlinearities, Control \& Cybernetics, to appear. 
[19] E. Zuazua, Some problems and results on the controllability of partial differential equations, in Progress in Mathematics, Proceedings of the Second European Congress of Mathematics, vol. 169, Birkhäuser Verlag, Basel/Switzerland, 1998, pp. 276-311. 